\title{
Validation of Salivary Cortisol among Filipino Adults Suspected with Cushing's Syndrome
}

\author{
Tom Edward N. Lo, Queenie G. Ngalob, Margarita Victoria Holgado-Galicia and Frances Lina Lantion-Ang \\ Section of Endocrinology, Diabetes and Metabolism, Department of Medicine, \\ College of Medicine and Philippine General Hospital, University of the Philippines Manila
}

\begin{abstract}
Background. Cushing's syndrome is a state of hypercortisolism manifesting non-specific clinical features where its diagnosis entails biochemical confirmation of cortisol excess. This study aims to validate the efficacy of midnight salivary cortisol as a screening test for Filipinos suspected with Cushing's syndrome and determine the cut-off value applicable in the local setting.
\end{abstract}

Methods. This is a cross-sectional study of Filipinos suspected with endogenous Cushing's syndrome seen at a tertiary hospital. Modification of plasma cortisol measured by RIA was used to measure salivary cortisol. The sensitivity, specificity, positive predictive value, negative predictive value and area under the curve of the screening tests were estimated and compared using 48 hour low dose dexamethasone suppression test (LDDST) as the reference standard.

Results. The determined cut-off value ( $\geq 7.0 \mathrm{nmol} / \mathrm{L}$ ) for salivary cortisol showed a relatively high sensitivity (91.3\%) and specificity $(89.5 \%)$ in detecting cases suspected of Cushing's syndrome. One milligram (1 $\mathrm{mg}$ ) dexamethasone suppression test had the highest sensitivity (100\%) but had the lowest specificity $(68.4 \%)$ as a screening test. The area under the curve of the three diagnostic tests appeared to be similar when compared with the low dose dexamethasone suppression test.

Conclusion. Using a cut-off value of $7 \mathrm{nmol} / \mathrm{L}$, local utility of latenight salivary cortisol has a high sensitivity and specificity in detecting Cushing's syndrome. It has a similar efficiency with 24hour urine free cortisol and $1 \mathrm{mg}$ dexamethasone suppression test as a screening test for Cushing's syndrome. Salivary cortisol may be considered as a valid initial screening test for Filipinos suspected of Cushing's syndrome.

Key Words: salivary cortisol, Cushing's syndrome, serum cortisol, dexamethasone suppression test

Paper presented at the Seoul International Congress of Endocrinology and Metabolism (SICEM), April 30-May 3, 2015, Seoul, South Korea; won first place at the PGH Fellow's Research Forum 2014; Poster presented at the Gulf Chapter of the American Association of Clinical Endocrinologists Congress 2014, Abu Dhabi, UAE; poster won at the Asia Oceania Congress of Endocrinology (AOCE), Oct. 911, 2014, Cebu City, Philippines.

Corresponding author: Tom Edward N. Lo, MD

Section of Endocrinology, Diabetes and Metabolism

Department of Medicine

Philippine General Hospital

University of the Philippines Manila

Taft Avenue, Ermita, Manila 1000 Philippines

Telephone: +639175297751

Email: tomedwardlo@yahoo.com

\section{Introduction}

Cushing's syndrome is a state of hypercortisolism manifesting non-specific clinical features such as obesity, hypertension, and weakness. $^{1}$ Its diagnosis entails biochemical confirmation of cortisol excess, which may also be observed in pseudo-Cushing states, such as major depression, chronic alcoholism, type 2 diabetes mellitus, visceral obesity, and polycystic ovary syndrome. This poses a diagnostic challenge to clinicians, hence the need for screening tests to confirm the diagnosis. ${ }^{1-5}$

The loss of diurnal rhythm is considered to be the hallmark of Cushing's syndrome and distinguishes it from normal individuals, including those with pseudo-Cushing states. Among the current screening tests, measurement of midnight or late-night serum cortisol has been considered as a reliable way of making this distinction by demonstrating if there is preservation of the physiologic circadian rhythm of cortisol secretion. However, this method is inconvenient, costly, and requires hospitalization to obtain the adequate serum sample. ${ }^{1-2}$

Raff et al have demonstrated the use of late-night salivary cortisol as an alternative to midnight serum cortisol measurement in screening patients for Cushing's syndrome. ${ }^{2}$ This has led to similar studies validating the usefulness of this technique as well. In Japan, Doi et al confirmed the reliability of late-night salivary cortisol measurement as a screening tool for patients with Cushing's syndrome and further extends its practical applicability as an initial screening test for subclinical Cushing's syndrome in highrisk populations, such as those with metabolic syndrome, obesity, and diabetes. ${ }^{4}$

Different techniques for measurement of salivary cortisol include radioimmunoassay (RIA), electrochemiluminescence immunoassay (ECLIA), tandem mass spectrometry (LC-MS/MS), and enzyme immunoassay (EIA). Of these methods, only RIA has been validated against plasma cortisol and urine free cortisol levels. ${ }^{1-5}$

The Endocrine Society has recommended in its clinical practice guidelines that screening tests for Cushing's syndrome include 24-hour urine free cortisol (UFC), lowdose dexamethasone suppression test (LDDST), or measurement of late-night salivary cortisol. ${ }^{6}$ The first two methods, however, may be cumbersome and expensive and give some degree of inaccuracy if specimen collection is not 
adequate. Moreover, confounding factors may give a significant rate of false positive and false negative results, such as intake of substances that induce cytochrome P450related enzymes or renal or hepatic failure. ${ }^{1}$

Late-night salivary cortisol determination is currently used as a first-line screening test in the US and several European countries. ${ }^{4}$ It reflects the unbound, biologically active form of serum cortisol and is independent of the rate of saliva production. ${ }^{1-2}$ It offers the advantages of high diagnostic accuracy (92-100\% sensitivity and $93-100 \%$ specificity) and convenient self-collection at home, making it an ideal diagnostic test suitable for screening even on an outpatient basis. ${ }^{4-6}$

Measurement of salivary cortisol is currently not available in the Philippines. This will be the first local study to demonstrate the modified technique for determination of salivary cortisol levels among Filipinos, results of which may validate its efficacy and usefulness in the local setting.

\section{Objectives}

1. To determine the accuracy of late night salivary cortisol in detecting Cushing's syndrome compared with LDDST (sensitivity, specificity, positive predictive value and negative predictive value).

2. To compute for the cut off value for salivary cortisol which is most predictive of Cushing's syndrome using a receiver operator curve (ROC).

\section{Methods}

\section{Participant Recruitment}

A cross-sectional criterion referenced study was done. Filipino patients seen at the Philippine General Hospital 18 years old and above, suspected with Cushing's syndrome based on suggestive signs and symptoms (e.g. dorsocervical fat pad, facial fullness, supraclavicular fullness, obesity, hirsutism or female balding, easy bruising, striae, peripheral edema) or based on presence of concomitant conditions (e.g. osteoporosis in the young, hypertension in the young, type 2 diabetes in the young, polycystic ovary syndrome, incidental adrenal mass, hypokalemia). Patients on exogenous intake of steroids, acutely ill or with on-going infection, pregnant, with intake of oral contraceptive pills within the past 6 weeks or anti-psychotic drugs, or have undergone dental procedure within the last 48 hours, were excluded.

Informed consent was obtained. All subjects underwent standardized measurements for height and weight, as well as a complete physical examination.

\section{Sample Size Calculation}

Due to difficulty in recruiting patients with suspected Cushing's syndrome, margin of error was set at $7 \%$. Based on a study by Raff, et al, which yielded $97 \%$ sensitivity and $95 \%$ specificity using a cut-off value of $2.7 \mathrm{nmol} / \mathrm{L}$, a sample size of 61 subjects (38 normal and 23 with suspicion of Cushing's syndrome) will yield similar results.

\section{Specimen Collection}

Saliva collection was done on an out-patient basis and written standardized instructions on proper sample collection were given to each subject. Each subject was given two (2) plain tubes for collection of saliva to be done at $11 \mathrm{pm}$ on two consecutive nights. Saliva was collected by bending the head forward, allowing saliva to drip into the tube until $1 \mathrm{ml}$ has been obtained. The tube was then capped and refrigerated $\left(4^{\circ} \mathrm{C}\right)$ and immediately sent to the Radioisotope Laboratory of the Philippine General Hospital within 24 hours of collection, for analysis.

\section{Determination of salivary cortisol}

Modification of plasma cortisol measured by RIA (Raff, et $\mathrm{al}^{3}$ ) was used to measure salivary cortisol. The assay was performed at the Radioisotope Laboratory of the Philippine General Hospital. This modified technique consisted of increasing analyte volume from 25 to $200 \mathrm{uL}$, increasing incubation time from 45 to 180 minutes, decreasing incubation temperature from $37 \mathrm{C}$ to room temperature, and diluting provided calibrators 1:10 in distilled water.

\section{Other laboratory tests done}

If not yet done by the referring physician, a 24-hour urine free cortisol, overnight $1 \mathrm{mg}$ dexamethasone suppression test, and low-dose dexamethasone suppression test (LDDST) were done on an out-patient basis as well. The LDDST was considered as the reference standard in this study.

\section{Data Analysis}

Data analysis was done using Stata SE version 12. Quantitative variables were summarized and presented as mean and standard deviation, while qualitative variables were tabulated and presented as frequency and percent distribution. Intra-rater reliability in measuring salivary cortisol level was tested by comparing the results of the first and second samples from each patient. The cut-off value of salivary cortisol was determined based on the graph of its sensitivity and specificity with LDDST as the reference standard. The sensitivity, specificity, positive predictive value, negative predictive value and area under the curve of salivary cortisol, 24 hour urine free cortisol and $1 \mathrm{mg}$ dexamethasone suppression test in detecting true endogenous hypercortisolism among patients suspected of Cushing's syndrome were estimated with 95\% confidence level. The area under the curve of the three diagnostic tests was compared to check for statistical difference with LDDST as the reference standard. The level of significance was set at 0.05 .

\section{Results}

A total of 61 participants suspected with Cushing's Syndrome were included in this study. Most were young and predominantly female. Abnormal fat distribution (i.e., 
moon facies, buffalo hump, and supraclavicular fat pad) appeared to be the most commonly observed clinical feature for both pseudo-cushing's and true Cushing's syndrome. Hypertension in the young, incidental adrenal mass and hypokalemia were the commonly associated co-morbidities for suspecting Cushing's syndrome in the absence of typical cushingoid features (Table 1).

Table 1. Baseline Characteristics of Study Participants Suspected of Endogenous Cushing's Syndrome

\begin{tabular}{|c|c|c|c|}
\hline & \multicolumn{2}{|c|}{ Non-Cushing's $(\mathrm{N}=38)$} & \multirow{2}{*}{$\begin{array}{c}\text { Cushing's } \\
\text { Syndrome } \\
(\mathrm{N}=23)\end{array}$} \\
\hline & $\begin{array}{l}\text { Normal } \\
(N=27)\end{array}$ & $\begin{array}{l}\text { Pseudo- } \\
\text { cushing's } \\
(\mathrm{N}=11)\end{array}$ & \\
\hline Age (years) & $43 \pm 12$ & $37 \pm 12$ & $35 \pm 13$ \\
\hline Female Sex (\%) & $21(77.8)$ & $10(90.9)$ & $18(78.3)$ \\
\hline Weight $(\mathrm{kg})$ & $59.6 \pm 16.3$ & $81.2 \pm 20.3$ & $63.4 \pm 12.4$ \\
\hline Height $(\mathrm{cm})$ & $156.9 \pm 7.7$ & $156.4 \pm 6.6$ & $156.2 \pm 10$ \\
\hline BMI $\left(\mathrm{kg} / \mathrm{cm}^{2}\right)$ & $24.1 \pm 6.3$ & $33.0 \pm 7.2$ & $25.9 \pm 3.9$ \\
\hline Systolic BP (mmHg) & $143 \pm 35$ & $139 \pm 18$ & $148 \pm 21$ \\
\hline Diastolic BP(mmHg) & $89 \pm 16$ & $90 \pm 8$ & $95 \pm 13$ \\
\hline \multicolumn{4}{|c|}{ Physical Findings Suggestive of Cushing's Syndrome } \\
\hline Buffalo Hump & 0 & $9(81.8)$ & $18(78.3)$ \\
\hline Moon Facies & 0 & $4(36.4)$ & $19(82.6)$ \\
\hline Supraclavicular Fat Pad & 0 & $9(81.8)$ & $17(73.9)$ \\
\hline Easy Bruisability & 0 & $1(9.1)$ & $10(43.5)$ \\
\hline Abdominal Striae & 0 & $3(27.3)$ & $11(47.8)$ \\
\hline Hirsutism & 0 & $3(27.3)$ & $12(52.2)$ \\
\hline Female Balding & 0 & 0 & $2(8.7)$ \\
\hline Peripheral Edema & 0 & $3(27.3)$ & $9(39.1)$ \\
\hline Acne & 0 & 0 & $4(17.4)$ \\
\hline \multicolumn{4}{|l|}{ Associated Co-morbidities } \\
\hline Hypertension & $16(59.3)$ & $7(63.6)$ & $20(87.0)$ \\
\hline Type 2 DM & $5(18.5)$ & $7(63.6)$ & $6(26.1)$ \\
\hline Osteoporosis & 0 & 0 & $1(4.3)$ \\
\hline PCOS & $6(22.2)$ & $3(27.3)$ & $2(8.7)$ \\
\hline Incidental Adrenal Mass & $15(55.6)$ & 0 & $6(26.1)$ \\
\hline Hypokalemia & $8(29.6)$ & $1(9.1)$ & $7(30.4)$ \\
\hline Obesity & $3(11.1)$ & $10(90.9)$ & $12(52.2)$ \\
\hline
\end{tabular}

Using a paired T-test, the salivary cortisol levels between the 2 samples taken from each patient were similar, thus there was no significant intra-rater variability (Table 2 ). Based on the graph of its sensitivity and specificity with LDDST as the reference standard, the cut-off value for salivary cortisol was determined to be at $7.0 \mathrm{nmol} / \mathrm{L}$. This value was derived from the intersection of the sensitivity and specificity curves (Figure 1). The outcomes of salivary cortisol, 24-hour urine free cortisol and 1mg dexamethasone suppression test against the LDDST are shown with a 2x2 table (Tables 3-5).

Based on the results, subjects were divided into three groups: normal (no signs and symptoms suggestive of Cushing's syndrome and normal LDDST), pseudo-Cushing's (signs and symptoms suggestive of Cushing's syndrome but normal LDDST), and Cushing's syndrome (abnormal LDDST with or without suggestive signs and symptoms).

The determined cut-off value ( $\geq 7.0 \mathrm{nmol} / \mathrm{L})$ for salivary cortisol showed a relatively high sensitivity and specificity in detecting cases suspected of Cushing's syndrome.
Compared to the 24-hour urine free cortisol, the sensitivity of salivary cortisol was almost similar while its specificity was calculated to be higher. One milligram (1 mg) dexamethasone suppression test had the highest sensitivity but had the lowest specificity as a screening test (Table 6). The area under the curve of the three diagnostic tests appeared to be similar when compared with the low dose dexamethasone suppression test (Table 7, Figure 2).

Table 2. Paired T-test Comparing Saliva Samples in Measuring Cortisol Levels

\begin{tabular}{ccccc}
\hline $\begin{array}{c}\text { Salivary } \\
\text { Sample }\end{array}$ & N & Mean & $\begin{array}{c}\text { Standard } \\
\text { Error }\end{array}$ & $\begin{array}{c}\text { Standard } \\
\text { Deviation }\end{array}$ \\
\hline $\mathbf{1}$ & 61 & 11.80 & 2.28 & 17.85 \\
$\mathbf{2}$ & 61 & 11.32 & 2.31 & 18.10 \\
Difference & 61 & 0.48 & 0.76 & 5.97 \\
& & & & T Score $=\mathbf{0 . 6 3 2 7}$ \\
\hline
\end{tabular}

Table 3. Salivary Cortisol vs. Low-dose Dexamethasone Suppression Test in Patients Suspected to have Cushing's Syndrome

\begin{tabular}{ccc}
\hline & \multicolumn{2}{c}{ Low-dose Dexamethasone Suppression Test } \\
\cline { 2 - 3 } Salivary Cortisol & Suppressed $(\mathrm{N}=38)$ & Unsuppressed $(\mathrm{N}=23)$ \\
\hline$<7.0 \mathrm{nmol} / \mathrm{L}(\mathrm{N}=36)$ & 34 & 2 \\
$\geq 7.0 \mathrm{nmol} / \mathrm{L}(\mathrm{N}=25)$ & 4 & 21 \\
\hline
\end{tabular}

Table 4. Urine free cortisol vs. Low-dose Dexamethasone Suppression Test

\begin{tabular}{ccc}
\hline & \multicolumn{2}{c}{ Low-dose Dexamethasone Suppression Test } \\
\cline { 2 - 3 } 24-hour UFC & Suppressed $(\mathrm{N}=38)$ & Unsuppressed $(\mathrm{N}=23)$ \\
\hline Normal $(\mathrm{N}=33)$ & 31 & 2 \\
Elevated $(\mathrm{N}=28)$ & 7 & 21 \\
\hline
\end{tabular}

Table 5. Overnight $1 \mathrm{mg}$ Dexamethasone Suppression Test vs. Low-dose Dexamethasone Suppression Test

\begin{tabular}{ccc}
\hline 1 mg Dexamethasone & \multicolumn{2}{c}{ Low-dose Dexamethasone Suppression Test } \\
\cline { 2 - 3 } Suppression Test & Suppressed $(\mathrm{N}=38)$ & Unsuppressed $(\mathrm{N}=23)$ \\
\hline Suppressed $(\mathrm{N}=26)$ & 26 & 0 \\
Unsuppressed $(\mathrm{N}=35)$ & 12 & 23 \\
\hline
\end{tabular}

Table 6. Sensitivity, Specificity, and Predictive Values of Screening Tests for Cushing's Syndrome

\begin{tabular}{lccc}
\hline & $\begin{array}{c}\text { Salivary } \\
\text { Cortisol }\end{array}$ & $\begin{array}{c}\text { 24-hr Urine } \\
\text { Free } \\
\text { Cortisol }\end{array}$ & $\begin{array}{c}\mathbf{1 ~ m g} \\
\text { Dexamethasone } \\
\text { Suppression } \\
\text { Test }\end{array}$ \\
\hline Sensitivity & 91.30 & 91.30 & 100.0 \\
{$[95 \% \mathrm{CI}]$} & {$[71.96-98.93]$} & {$[71.96-98.93]$} & {$[85.18-100.0]$} \\
Specificity & 89.47 & 81.58 & 68.42 \\
{$[95 \% \mathrm{CI}]$} & {$[75.20-97.06]$} & {$[65.67-92.26]$} & {$[51.35-82.50]$} \\
Positive Predictive & 84.00 & 75.00 & 65.71 \\
Value [95\% CI] & {$[63.92-95.46]$} & {$[55.13-89.31]$} & {$[47.79-80.87]$} \\
Negative Predictive & 94.44 & 93.94 & 100.0 \\
Value [95\% CI] & {$[81.34-99.32]$} & {$[79.77-99.26]$} & {$[86.77-100.0]$} \\
\hline
\end{tabular}


Table 7. Comparison of the Area under the Curve of 3 Diagnostic Procedures as Screening Test

\begin{tabular}{lccc}
\hline \multicolumn{1}{c}{ Diagnostic Test } & $\begin{array}{c}\text { Area Under } \\
\text { the Curve }\end{array}$ & $\begin{array}{c}\mathbf{9 5 \%} \text { Confidence } \\
\text { Interval }\end{array}$ & $\begin{array}{c}\text { p-Value } \\
<\mathbf{0 . 0 5}\end{array}$ \\
\hline Midnight Salivary Cortisol & 0.9668 & $0.9208-1.0$ & 0.0810 \\
24-hour Urine Free Cortisol & 0.9445 & $0.8854-1.0$ & \\
1 mg Dexamethasone & 0.9977 & $0.9922-1.0$ & \\
Suppression Test & & & \\
\hline
\end{tabular}

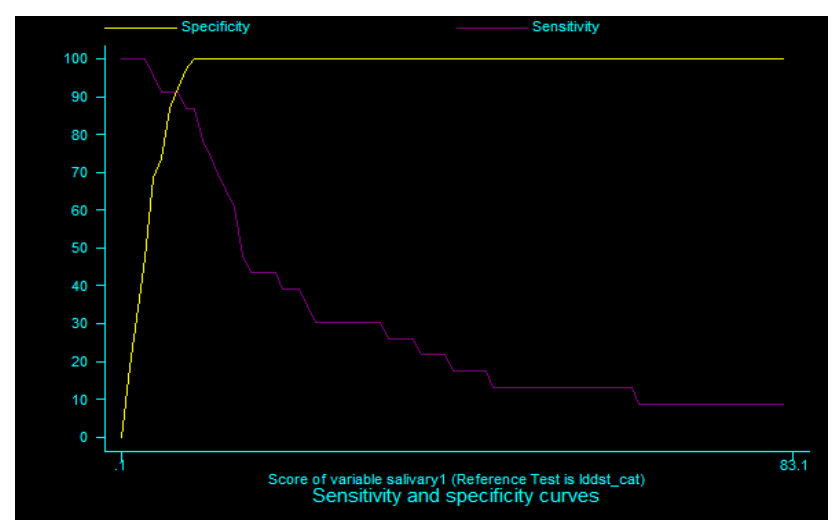

Figure 1. Sensitivity and Specificity Curve of Salivary Cortisol in Detecting Cushing's Syndrome

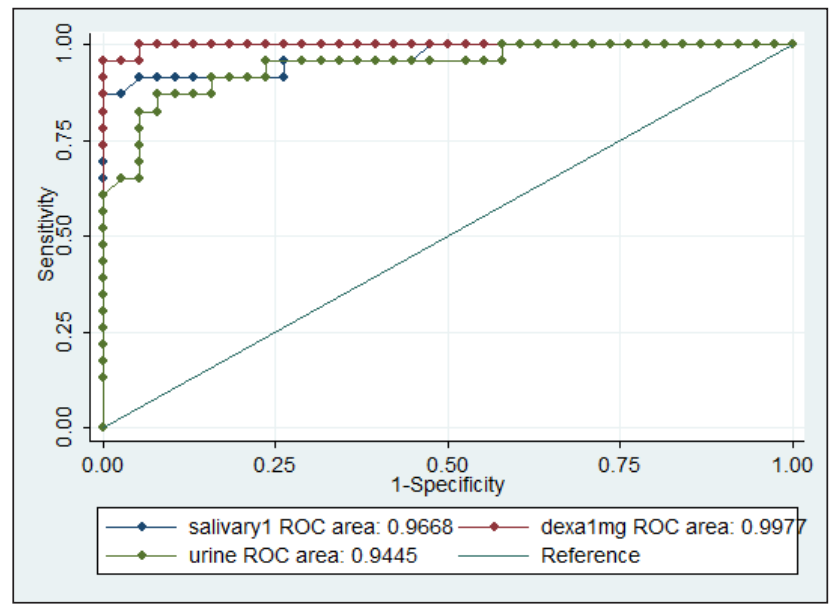

Figure 2. Comparison of the Area under the Curve of 3 Diagnostic Tests as Screening for Patients with Cushing's Syndrome

\section{Discussion}

Based on the results of this study, late-night salivary cortisol may be considered as an accurate screening test for Filipinos with suspected Cushing's syndrome. This was shown by the similarity of the area under the curve of the three diagnostic tests (1 mg DST, $24 \mathrm{hr}$ UFC, midnight salivary cortisol) with the reference standard (LDDST).

The applicability of this test in the local setting offers several advantages especially to patient's convenience. Salivary cortisol measurements reflect the unbound fraction of circulating cortisol which is unaffected by cortisol-binding globulins. It is also unaffected by saliva flow rates. ${ }^{1-2}$ Sample collection done at home lowers medical costs, and lessen patient's anxiety and discomfort leading to better patient's confidence with the test. However, clear description of the standard sample collection procedure must be provided to patients as confusion with sample collection procedures might lead to collection errors and stressors to the patient. ${ }^{1}$

Similar studies presented different cut-off values that gave highly sensitive and specific results in screening Cushing's syndrome (Table 8). It is important to set locally validated cut-off values rather than adopting cut-off points established by previous studies done in other populations.

Table 8. Different Cut-off Points of Late Night Salivary Cortisol Validated by Different Validation Studies

\begin{tabular}{lccc}
\hline Validation Studies & $\begin{array}{c}\text { Cut-off Values } \\
\text { (nmol/L) }\end{array}$ & Sensitivity & Specificity \\
\hline Present Study & 7.0 & 91.3 & 89.5 \\
Putignano et.al. $^{5}$ & 9.7 & 92.7 & 93.1 \\
Papanicolaou et.al. $^{1}$ & 15.2 & 93.0 & 100.0 \\
Raff et.al. $^{2}$ & 2.7 & 97.0 & 95.0 \\
\hline
\end{tabular}

The similar accuracy of the late-night salivary cortisol as a screening test makes it a good additional test in the armamentarium of diagnostics in establishing the diagnosis of Cushing's syndrome. Salivary cortisol is a promising test in our local setting.

\section{Conclusion}

Using a cut-off value of $7 \mathrm{nmol} / \mathrm{L}$, the local utility of late-night salivary cortisol has a high sensitivity and specificity in detecting Cushing's syndrome. It has a similar accuracy with 24-hour urine free cortisol and 1mg dexamethasone suppression test and may be used as a screening test for Cushing's syndrome. Salivary cortisol is a valid initial screening test for Filipinos suspected of Cushing's syndrome.

\section{References}

1. Papanicolaou DA, Mullen N, Kyrou I, Nieman LK. Nighttime salivary cortisol: a useful test for the diagnosis of Cushing's syndrome. J Clin Endocrinol Metab. 2002, 87(10):4515-21.

2. Raff H, Raff JL, Findling JW. Late-night salivary cortisol as a screening test for Cushing's syndrome. J Clin Endocrinol Metab 1998; 83(8):2681-6.

3. Vilar L, Freitas MC, Naves LA, et al. The role of non-invasive dynamic tests in the diagnosis of Cushing's syndrome. J Endocrinol Invest. 2008; 31(11):1008-13.

4. Doi M, Sekizawa N, Tani Y, et al. Late-night salivary cortisol as a screening test for the diagnosis of Cushing's syndrome in Japan. Endocr J. 2008; 55(1):121-6.

5. Putignano P, Toja P, Dubini A, Pecori Giraldi F, Corsello SM, Cavagnini F. Midnight salivary cortisol versus urinary free and midnight serum cortisol as screening tests for Cushing's syndrome. J Clin Endocrinol Metab. 2003; 88(9):4153-7.

6. Nieman LK, Biller BM, Findling JW, et al. The diagnosis of Cushing's syndrome: an Endocrine Society Clinical Practice Guideline. J Clin Endocrinol Metab. 2008, 93(5):1526-40. 